\title{
The relation between latissimus dorsi skeletal muscle structure and contractile function after cardiomyoplasty
}

Past reports suggest that structural changes within the latissimus dorsi muscle occur with chronic electrical stimulation during cardiomyoplasty. However, the specific changes in the structure of the latissimus dorsi muscle and the relation to muscle contractile function with cardiomyoplasty are unknown. Accordingly, this study examined regional changes in latissimus dorsi muscle structure and function after cardiomyoplasty. The left latissimus dorsi muscle was mobilized and wrapped around the heart in pigs with the use of standardized techniques and the latissimus dorsi muscle chronically paced at ambient heart rates ( 90 beats/min; $20 \mathrm{~Hz}, 5 \mathrm{~V}$ amplitude, $n=6$ ). After 6 weeks, the paced latissimus dorsi muscle and the contralateral control muscle were removed and divided into proximal $(0$ to $3 \mathrm{~cm}$ ), middle $(3$ to $6 \mathrm{~cm}$ ), and distal $(6$ to $12 \mathrm{~cm}$ ) regions. By computer-assisted morphometry, muscle cell myofibril volume, cross-sectional area, and collagen percent area were determined. In the paced latissimus dorsi muscle, myofibril volumes increased by more than $50 \%$ in the proximal and middle regions compared with those in the contralateral control muscle. However, myofibril volumes were significantly lower in the distal region of the paced latissimus dorsi muscle compared with those in control muscles $(33 \% \pm 5 \%$ versus $20 \% \pm 3 \%, p<0.05)$. In the paced latissimus dorsi muscle, cross-sectional area was significantly reduced from that of control muscles in all regions. $A$ further reduction in cross-sectional area was noted in the distal region of the paced latissimus dorsi muscle compared with that in both the contralateral control muscle and the proximal and middle regions of the paced latissimus dorsi muscle. Collagen content significantly increased in the paced latissimus dorsi muscle compared with that in control muscle with a more fibrotic pattern observed in the distal region. Latissimus dorsi muscle strips (less than $2 \mathrm{~mm}^{2}$ cross-sectional area) were harvested, and peak and velocity of tension development were examined after field electrical stimulation at 0.2 to $1.2 \mathrm{~Hz}$. At 0.2 $\mathrm{Hz}$, the velocity of tension development was unchanged in the paced latissimus dorsi muscle compared with that in control muscle. However, peak tension development degraded by only $28 \%$ in the paced latissimus dorsi muscles but fell by $51 \%$ in control muscles with increased stimulation frequencies. In summary, the contractile function of the chronically stimulated latissimus dorsi muscle was associated with fatigue resistance and increased contractile protein content. However, more distal regions of the paced latissimus dorsi muscle demonstrated atrophy and fibrosis. These region-specific changes within the chronically stimulated latissimus dorsi muscle may significantly influence the long-term performance of this skeletal muscle after cardiomyoplasty. (J THORAC CARDIOvasC SURG 1994;107:868-78)

John M. Kratz, MD, Wendy S. Johnson, LVT, Rupak Mukherjee, MS, Jie Hu, MD, Fred A. Crawford, MD, and Francis G. Spinale, MD, PhD, Charleston, S.C.

From the Division of Cardiothoracic Surgery, Department of Surgery, Medical University of South Carolina, Charleston, S.C.

Supported in part by National Institutes of Health grant HL45024 (F.G.S.) and Medtronic, Inc., Minneapolis, Minn.

Received for publication June 9, 1993.

Accepted for publication Aug. 30, 1993.
Address for reprints: John M. Kratz, MD, Division of Cardiothoracic Surgery, Medical University of South Carolina, 171 Ashley Ave., Charleston, SC 29425.

Copyright $\odot 1994$ by Mosby-Year Book, Inc.

$0022-5223 / 94 \$ 3.00+0 \quad 12 / 1 / 51202$ 
Dynamic cardiomyoplasty has recently been suggested to be an effective method for the surgical treatment of patients with end-stage heart failure. ${ }^{1-4}$ By special electrical pacing techniques, the latissimus muscle is trained with the conversion of fast-twitch fibers to fatigue-resistant slow-twitch fibers. ${ }^{5-9}$ Dynamic cardiac assistance is then provided by pacing the latissimus muscle in synchrony with the intrinsic contractions of the ventricle..$^{1-6}$ A large number of patients have been surgically treated with dynamic cardiomyoplasty worldwide and have demonstrated improvements in clinical symptoms of heart failure. ${ }^{1-4}$ However, the consequences of chronic electrical stimulation of the latissimus dorsi muscle (LDM) during dynamic cardiomyoplasty remain unclear. For cardiomyoplasty to be considered as a viable therapy for treatment of patients with end-stage heart failure, the changes in structure and function of the LDM after cardiomyoplasty must be clearly understood. Thus the overall goal of this project was to determine the specific regional changes in structure and function that occur within the LDM after dynamic cardiomyoplasty.

\section{Methods}

Cardiomyoplasty procedure. Six Yorkshire swine ( 20 to 30 $\mathrm{kg}$ ) were anesthetized with isoflurane $(3 \% / 1.5 \mathrm{~L} / \mathrm{min})$ and nitrous oxide $(0.5 \mathrm{~L} / \mathrm{min})$, intubated with a cuffed endotracheal tube, and the lungs ventilated at a flow rate of $22 \mathrm{ml} / \mathrm{kg}$ per minute and a respiratory rate of 15 breaths $/ \mathrm{min}$. The animals were placed in a left lateral recumbency and the left LDM exposed through an incision along the short axis of the muscle. The muscle was dissected free from all insertions and the humoral tendon was cut. Great care was taken to preserve the thoracodorsal neurovascular pedicle. A platinum nerve cuff stimulating electrode (Medtronic, Inc., Minneapolis, Minn.) was positioned around the origin of the thoracodorsal nerve and sutured in place. Next, a thoracotomy was done at the fourth intercostal space and a window was created by resection of the lateral part of the third rib. The mobilized LDM was introduced through this window and positioned within the chest. Careful inspection was done at this time to ensure that there was no compression or deformities of the thoracodorsal neurovascular pedicle or nerve-stimulating electrode. The pericardium was excised widely and the muscle was wrapped around the circumference of the heart. The LDM was carefully positioned to be parallel with the short axis of the left ventricle and sutured to the epicardial surface of the myocardium. A shielded electrode was sutured on the epicardial surface of the left ventricle for sensing ventricular depolarization. ${ }^{10}$ The nerve-stimulating electrode and the sensing electrode were connected to a myostimulator unit (SP1005, Medtronic). The thoracotomy was then closed, and the pleural space evacuated of air. The animal was then allowed to recover for 14 days before the stimulation protocol was begun.

After recovery from the surgical procedure, the muscle was stimulated with incremental increases in pulse duration and frequency following a protocol that has been fully described previously. ${ }^{1-7,11}$ The final stimulation settings for this project were a pulse amplitude of $1.5 \mathrm{~V}$, pulse duration of $210 \mathrm{msec}$, frequency of $20 \mathrm{~Hz}$, and frequency burst of $185 \mathrm{msec}$. The muscle underwent chronic electrical stimulation for 6 weeks. Previous studies have clearly demonstrated that these burst frequency and pulse training characteristics successfully prepared the muscle for dynamic assist. ${ }^{1-6,11}$ An electrocardiogram was obtained on a weekly basis throughout the muscle stimulation protocol to ensure continuous operation of the muscle stimulator. All animals were treated and cared for in accordance with the National Institutes of Health "Guide for the Care and Use of Laboratory Animals."12

Before the initiation of electrical stimulation of the LDM, echocardiographic measurements of left ventricular size and function were obtained as previously described. ${ }^{13}, 14$ Twodimensional and M-mode echocardiographic studies (ATL Ultramark VI, $2.25 \mathrm{MHz}$ transducer, Bothell, Wash.) were used to image the left ventricle from a right parasternal approach. Left ventricular fractional shortening was computed as (end-diastolic dimension - end-systolic dimension)/end-diastolic dimension and was expressed as a percentage. After the six-week stimulation protocol period and just before the terminal studies, these echocardiographic measurements were repeated.

Terminal study. After 6 weeks of chronic electrical stimulation of the LDM, the animals were returned to the laboratory and anesthetized as described in the previous section. The animal was placed in a dorsal recumbency, and the right LDM was exposed and removed. This contralateral, unstimulated muscle served as a control for each experiment. After removal, the LDM was immediately placed in chilled, oxygenated Krebs solution and prepared for structural analysis and function studies as described in the following sections. A sternotomy was then done, and the entire left LDM was carefully dissected free from the heart and placed in oxygenated Krebs solution.

Before preparation of the control and chronically stimulated muscles for structural studies, a minimum of six muscle strips were harvested from the midportion of each muscle and prepared for function studies as described in the following section. To examine regional differences in skeletal muscle structure, both the control and cardiomyoplasty muscle were divided into three regions: proximal, 0 to $4 \mathrm{~cm}$ from the origin of thoracodorsal artery; middle, 4 to $7 \mathrm{~cm}$ from the thoracodorsal artery; and distal, $7 \mathrm{~cm}$ and beyond from the origin of the thoracodorsal artery. Once both muscles had been divided into these regions, 3 by $3 \mathrm{~cm}$ cubes of muscle were taken from each of these three regions and prepared for light microscopy, 2 by $2 \mathrm{~cm}$ cubes were prepared for electron microscopy, and 3 by 3 $\mathrm{cm}$ cubes were flash-frozen in liquid nitrogen for subsequent hydroxyproline analysis.

Muscle fiber function. Muscle sections ( $2 \mathrm{~mm}$ outer diameter) were taken from the chronically stimulated and control LDMs and placed in an oxygenated Krebs solution. The muscle strips were mounted in a jacketed muscle chamber $(25 \mathrm{ml}$, $37^{\circ} \mathrm{C}$ ) with one end tied to a fixed post and the other to an isometric force transducer (Grass FT-03; Quincy, Mass.). After attachment, the muscle strips were stretched in $1 \mathrm{~mm}$ increments and the resultant passive tension monitored. Maximal length was defined as the length of the muscle strip at which the passive tension did not decay. ${ }^{15}$ Muscle twitches were elicited by electrical field stimulation. Electrical pulses were delivered to the muscle strips via two platinum ring electrodes placed on either side of the muscle strips. ${ }^{15}$ The duration of the stimuli 
generated by an electrical stimulator (Grass S5) was $10 \mathrm{msec}$ and stimulation voltage was maintained at $10 \%$ above the contraction threshold. For baseline measurements, stimulation frequency was maintained at $0.2 \mathrm{~Hz}$. Isometric tension was recorded by an isometric force transducer (Grass FT-03) whose output was amplified (Grass 7P1 A direct-current preamplifier) and recorded on a strip-chart recorder (Gould ES1000, Oxnard, Calif.). The analog tension signal was also digitized at a sampling frequency of $200 \mathrm{~Hz}$ (DT2801; Data Translation, Marlboro, Mass.) and the digitized data stored on disk.

Individual muscle fiber twitches were analyzed off-line for peak tension development, the velocity of tension development $(+\mathrm{dT} / \mathrm{dt})$, the velocity of tension relaxation $(-\mathrm{dT} / \mathrm{dt})$, the time to peak tension development, the time to $50 \%$ relaxation, and the total duration of the twitch. After the collection of tension data at baseline, the fatigue-resistant properties of the muscle strips were examined by increasing the stimulation frequency to 0.4 $\mathrm{Hz}, 0.8 \mathrm{~Hz}$, and $1.2 \mathrm{~Hz}$. Peak isometric tension was recorded at each frequency and averaged over a minimum of 20 consecutive twitches. The relative change in the amplitude of the twitches from baseline values was used as an index of muscle fatigue. After the stimulation protocol, the muscle fiber was cut in cross section and the cross-sectional area measured. Contraction profile parameters were then normalized to this measured crosssectional area. ${ }^{15}$

Light microsocpy. Tissue blocks taken from each region of the control and chronically stimulated LDMs were immersed in $10 \%$ buffered formalin overnight and then transferred to $70 \%$ ethanol. These tissue blocks were then cut into $4 \mathrm{~mm}$ full-thickness sections and processed through graded ethanols, cleared in xylenes, and embedded in paraffin (Paraplast). Slices of $4 \mu \mathrm{m}$ in thickness were cut from the blocks and mounted on glass slides and stained with hematoxylin and eosin for morphometric analysis. The cross-sectional area of muscle cells within the LDM was computed by methods previously described for cardiac muscle. ${ }^{13}$ The stained skeletal muscle sections were mounted on an inverted microscope (IM-35; Ziess, Munich, W Germany) and circumferentially oriented muscle fibers were imaged with an epifluorescence illuminator with a rhodamine filter at a magnification of $630 \times$. The image was then analyzed with a computer image analysis system (IBAS 2000 automatic image analysis system; Zeiss/Kontron, Oberkochen/Munich, Germany). Epifluorescence provided a high contrast between the muscle cells and the extracellular space, allowing for simple and reliable digitization of the cell profiles. Ten random fields of circumferentially oriented muscle cells were examined from each slide and digitized. The sample field area was $7600 \mu \mathrm{m}^{2}$ and the system was calibrated with a stage micrometer before each digitizing session. Three slices at 1,2 , and $3 \mathrm{~mm}$ deep from each tissue block were examined. By this method, nine full-thickness sections from the three regions of each muscle were examined. Thus a total of 27 sections were examined from the contralateral control and chronically stimulated LDMs from each pig.

To more carefully examine the distribution and content of extracellular fibrous tissue within the control and chronically stimulated LDMs, we analyzed tissue sections for the percent area occupied by collagen as described previously by this laboratory. ${ }^{14}$ Muscle sections were cut to $5 \mu \mathrm{m}$ thickness and stained by a silver-impregnation method. ${ }^{14}$ By computer-assisted morphometry, the stained sections were analyzed for the percent area of positive staining. ${ }^{14}$ The sections were imaged on an inverted microscope with a final magnification of $250 \times$. Morphometric measurements were done by the previously described image analysis system. The percent area of collagen was computed from 15 random fields excluding large vascular areas from each section. Mean collagen volume fraction was calculated by averaging the sum of all collagen areas and dividing it by the sum of all extracellular and muscle area and expressed as a percentage.

Electron microscopy. The muscle samples for electron microscopy were minced finely, immersed in a buffered sodium cacodylate solution containing $2 \%$ paraformaldehyde, $2 \%$ glu-

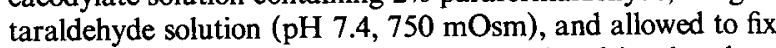
overnight. The tissue samples were then rinsed in phosphate buffer $0.1 \mathrm{~mol} / \mathrm{L}$, postosmicated for 1 hour in $1 \%$ osmium tetroxide, dehydrated in an ascending series of alcohols, and embedded in Epon resin. Thick sections were taken from these tissue blocks at a thickness of $1 \mu \mathrm{m}$, stained with toluidine blue, and viewed at $100 \times$ to obtain areas of the tissue blocks where myofibers were oriented in a circumferential direction. Six tissue blocks in the circumferential orientation from each of the three muscle regions were then used to obtain thin sections for electron microscopy. Three grids, containing three thin sections each, were prepared from each block. Thus a total of 54 sections were examined from each of the muscle regions taken from each pig. Thin sections were stained with uranyl acetate and lead citrate and examined with a JEOL $100 \mathrm{~S}$ electron microscope (JEOL USA, Inc., Peabody, Mass.). The central portion of each section was photographed at a calibrated magnification of $\times 10,000$. These electron micrographs were then coded and this code was not broken until the completion of the study. From the circumferentially oriented micrographs, the percent volume of myofibrils and mitochondria within the skeletal muscle cells was analyzed morphometrically with the use of a stereology sampling grid consisting of 140 sampling points. ${ }^{13}$

Collagen biochemistry. A biochemical assay for hydroxyproline, an amino acid found exclusively in collagen, was done on the previously frozen muscle sections by methods well. described previously. ${ }^{14,16}$ The muscle tissue was lyophilized to a constant weight, pulverized in the presence of dry ice, and relyophilized. The ground tissue was then hydrolyzed with $6 \mathrm{~N}$ hydrochloric acid at $107^{\circ} \mathrm{C}$ for 16 hours, resuspended in water, and decolorized with activated charcoal. The filtrate was diluted to a convenient volume and measured spectrophotometrically $(550 \mathrm{~nm})$ after reaction with Ehrlich's reagent. ${ }^{16}$ Results were expressed as milligrams hydroxyproline per gram dry weight tissue.

Data analysis. Comparisons of skeletal muscle function, muscle cell morphologic features, and collagen content between the control and cardiomyoplasty muscles were done by multiway analysis of variance. To determine regional differences in skeletal muscle structure with chronic electrical stimulation, skeletal fiber location was used as a fixed effect in an analysis of variance model. Changes in muscle fiber contractile performance as a function of stimulation frequency were computed as the percent difference from $0.2 \mathrm{~Hz}$ and comparisons made by analysis of variance. In the subproject in which comparisons of regional differences in muscle fiber function were done, the Wilcoxon test was used. ${ }^{17} \mathrm{~A}$ pilot study of three control LDMs in which the morphometric sampling techniques described in the previous section were used resulted in less than an $8 \%$ coefficient of variation for the parameters computed in this study. Before analysis of variance was done on the morphometric data, homogeneity of variances of each morphometric variable with respect to control and chronically stimulated LDM sections was confirmed by Bartlett's test. ${ }^{18}$ If the analysis of variance 
Table I. LDM contractile function after chronic stimulation

\begin{tabular}{|c|c|c|c|c|}
\hline & \multicolumn{4}{|c|}{ Stimulation frequency } \\
\hline & $0.2 \mathrm{~Hz}$ & $0.4 \mathrm{~Hz}$ & $0.8 \mathrm{~Hz}$ & $1.2 \overline{\mathrm{Hz}}$ \\
\hline \multicolumn{5}{|l|}{ Normalized peak tension $\left(\mathrm{gm} / \mathrm{cm}^{2}\right)$} \\
\hline Control LDM & $70.34 \pm 7.84$ & $57.12 \pm 6.34$ & $44.75 \pm 5.20^{*}$ & $32.28 \pm 3.54^{*}$ \\
\hline Chronically stimulated LDM & $51.13 \pm 7.51 \dagger$ & $44.73 \pm 6.05$ & $40.15 \pm 5.19$ & $35.78 \pm 4.39$ \\
\hline \multicolumn{5}{|c|}{ Normalized velocity of tension development $\left(\mathrm{gm} / \mathrm{sec} / \mathrm{cm}^{2}\right)$} \\
\hline Control LDM & $309.94 \pm 53.47$ & $250.54 \pm 45.56$ & $135.43 \pm 18.96^{*}$ & $94.73 \pm 13.23^{*}$ \\
\hline Chronically stimulated LDM & $237.62 \pm 51.73$ & $196.76 \pm 48.99$ & $145.12 \pm 36.66^{*}$ & $103.12 \pm 43.60^{*}$ \\
\hline \multicolumn{5}{|c|}{ Normalized velocity of tension relaxation $\left(\mathrm{gm} / \mathrm{sec} / \mathrm{cm}^{2}\right)$} \\
\hline Control LDM & $133.19 \pm 30.67$ & $113.54 \pm 27.21$ & $82.19 \pm 22.62$ & $46.28 \pm 11.38^{*}$ \\
\hline Chronically stimulated LDM & $75.33 \pm 15.00$ & $62.88 \pm 14.06 \dagger$ & $46.62 \pm 11.15^{* \dagger}$ & $33.23 \pm 6.82 * \dagger$ \\
\hline \multicolumn{5}{|l|}{ Time to peak tension (msec) } \\
\hline Control LDM & $81.23 \pm 3.00$ & $78.85 \pm 2.47$ & $73.95 \pm 3.51^{*}$ & $73.74 \pm 2.92^{*}$ \\
\hline Chronically stimulated LDM & $88.41 \pm 3.31$ & $86.82 \pm 3.04 \dagger$ & $84.32 \pm 2.43 \dagger$ & $84.07 \pm 1.73 \dagger$ \\
\hline \multicolumn{5}{|l|}{ Total twitch duration (msec) } \\
\hline Control LDM & $348.25 \pm 15.17$ & $318.41 \pm 18.45$ & $282.92 \pm 21.72^{*}$ & $263.71 \pm 20.67^{*}$ \\
\hline Chronically stimulated LDM & $381.43 \pm 9.24 \dagger$ & $366.87 \pm 11.04 \dagger$ & $349.87 \pm 12.00^{*} \uparrow$ & $339.23 \pm 11.48 * \dagger$ \\
\hline \multicolumn{5}{|l|}{ Time to $50 \%$ relaxation $(\mathrm{msec})$} \\
\hline Control LDM & $83.67 \pm 3.82$ & $80.03 \pm 3.39$ & $75.06 \pm 3.22^{*}$ & $71.81 \pm 3.47^{*}$ \\
\hline Chronically stimulated LDM & $96.55 \pm 3.59 \dagger$ & $90.23 \pm 3.44 \uparrow$ & $86.74 \pm 1.49^{* \dagger}$ & $89.93 \pm 3.84^{*} \dagger$ \\
\hline
\end{tabular}

All values presented as mean plus or minus standard error of the mean.

${ }^{*} p<0.05$ versus $0.2 \mathrm{~Hz}$

$t_{p}<0.05$ versus control LDM.

Table II. Regional changes in muscle fiber contractile function after chronic electrical stimulation of LDM with cardiomyoplasty

\begin{tabular}{lcc}
\hline & Proximal & Distal \\
\hline Normalized peak tension $\left(\mathrm{gm} / \mathrm{cm}^{2}\right)$ & $51.13 \pm 7.51$ & $39.00 \pm 11.56^{*}$ \\
Normalized velocity of tension development $\left(\mathrm{gm} / \mathrm{sec} / \mathrm{cm}^{2}\right)$ & $237.62 \pm 51.73$ & $103.12 \pm 43.60^{*}$ \\
Normalized velocity of tension relaxation $\left(\mathrm{gm} / \mathrm{sec} / \mathrm{cm}^{2}\right)$ & $75.33 \pm 15.00$ & $36.27 \pm 22.09^{*}$ \\
Time to peak tension $(\mathrm{msec})$ & $88.41 \pm 3.31$ & $95.69 \pm 2.98^{*}$ \\
Total twitch duration $(\mathrm{msec})$ & $381.43 \pm 9.24$ & $440.43 \pm 20.61^{*}$ \\
Time to $50 \%$ relaxation $(\mathrm{msec})$ & $96.55 \pm 3.59$ & $114.08 \pm 5.93^{*}$ \\
\hline
\end{tabular}

All values presented as mean plus or minus standard error of the mean. Stimulation frequency: $0.2 \mathrm{~Hz} ; n=\mathrm{two}$ pigs.

${ }^{*} p<0.05$ versus proximal, Wilcoxon test.

revealed significant differences, pairwise tests of individual group means were compared by Tukey's procedure. ${ }^{19}$ Results are presented as mean \pm standard error of the mean. Values of $p<0.05$ were considered to be statistically significant.

\section{Results}

All of the pigs used in this study completed the protocol and the contralateral control and chronically stimulated LDMs were successfully harvested for study. Before the initiation of electrical stimulation of the LDM, baseline left ventricular end-diastolic dimension was $3.5 \pm 0.3$ $\mathrm{cm}$ and fractional shortening was $31 \% \pm 3 \%$. After the 6-week stimulation protocol, the synchronous left ventricular and LDM contractions resulted in a significantly increased left ventricular fractional shortening $(39 \% \pm$ $2 \%, p<0.05)$ from baseline values with no significant change in end-diastolic dimension $(3.3 \% \pm 0.3 \%, p<$
0.75). At terminal study, successful stimulation of the chronically stimulated muscle was visually confirmed in all of the surgical preparations before harvest. For the contralateral control muscle, the expired time from dividing the thoracodorsal neuromuscular pedicle and placing muscle cubes into fixative and liquid nitrogen was $5 \pm 2$ minutes. For the chronically stimulated muscle, the time required for preparation of the muscle samples after interruption of the neurovascular pedicle was $6 \pm 2$ minutes and was not significantly different from that of the control harvest $(p>0.70)$.

Skeletal muscle function. A minimum of six muscle fiber samples each were taken from the control and chronically stimulated LDMs of each pig. Representative contraction profiles from a muscle fiber taken from a control and chronically stimulated LDM are shown in 

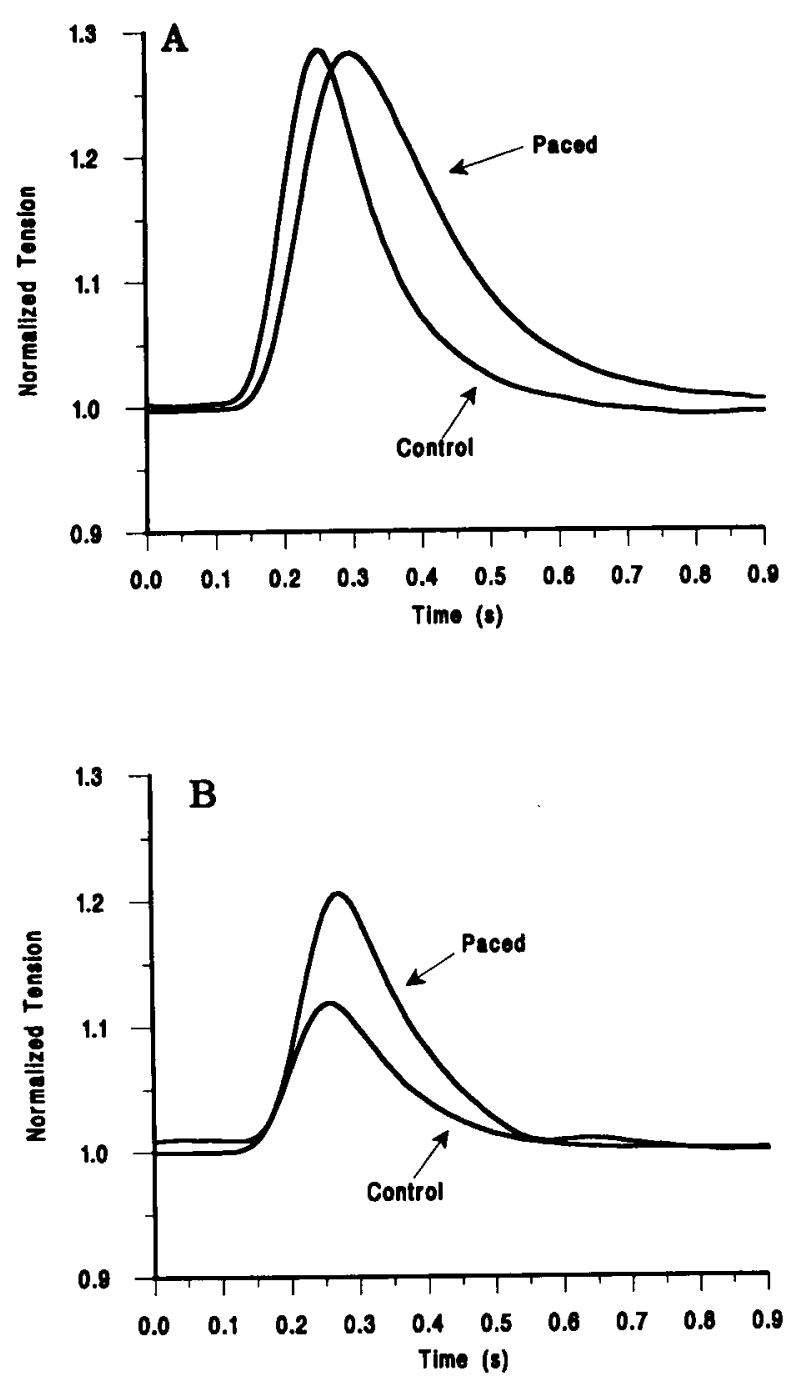

Fig. 1. A, Representative contraction profiles from chronically stimulated LDM fibers (Paced) and from contralateral control muscle. These contraction profiles were obtained using stimulation frequency of $0.2 \mathrm{~Hz}$. Chronically stimulated LDM demonstrated slightly reduced normalized peak tension development with prolonged twitch duration. B, At $1.2 \mathrm{~Hz}$, normalized velocity of tension development was higher in chronically stimulated LDM fibers compared with that in contralateral control fibers. In addition, time to peak tension significantly increased from control values. Summary of muscle function data obtained in this portion of study is presented in Table I.

Fig. 1. At $0.2 \mathrm{~Hz}$ stimulation frequency, the chronically stimulated muscle fiber displayed a significantly prolonged contraction profile compared with that of the control muscle (Fig. 1, A). At $1.2 \mathrm{~Hz}$ stimulation frequency, the velocity of tension development was higher in the chronically stimulated muscle compared with that of the control muscles (Fig. 1, B). A summary of the contraction data obtained from muscle fibers taken from the
Table III. Regional changes in LDM structure and biochemical composition after chronic stimulation

\begin{tabular}{|c|c|c|}
\hline & Control & Chronically stimulated \\
\hline \multicolumn{3}{|c|}{ Muscle cell cross-sectional area $\left(\mu \mathrm{m}^{2}\right)$} \\
\hline Proximal region & $1528 \pm 56$ & $1110 \pm 38^{*}$ \\
\hline Middle region & $1813 \pm 48$ & $1378 \pm 42^{*}$ \\
\hline Distal region & $1883 \pm 52$ & $836 \pm 47 * \dagger$ \\
\hline \multicolumn{3}{|c|}{ Myofibril percent volume (\%) } \\
\hline Proximal region & $30 \pm 3$ & $48 \pm 5^{*}$ \\
\hline Middle region & $32 \pm 4$ & $44 \pm 3^{*}$ \\
\hline Distal region & $33 \pm 4$ & $20 \pm 3^{* \dagger}$ \\
\hline \multicolumn{3}{|c|}{ Mitochondrial percent volume (\%) } \\
\hline Proximal region & $12 \pm 3$ & $20 \pm 5^{*}$ \\
\hline Middle region & $15 \pm 4$ & $23 \pm 4^{*}$ \\
\hline Distal region & $16 \pm 5$ & $30 \pm 4^{* \dagger}$ \\
\hline \multicolumn{3}{|c|}{ Collagen percent area $(\%)$} \\
\hline Proximal region & $5 \pm 2$ & $9 \pm 2^{*}$ \\
\hline Middle region & $6 \pm 2$ & $14 \pm 2^{*}$ \\
\hline Distal region & $5 \pm 2$ & $18 \pm 3^{*}$ \\
\hline \multicolumn{3}{|c|}{ Hydroxyproline content (mg/gdwt) } \\
\hline Proximal region & $3.2 \pm 0.5$ & $7.1 \pm 0.5^{*}$ \\
\hline Middle region & $4.2 \pm 0.6$ & $8.6 \pm 1.1^{*}$ \\
\hline Distal region & $5.0 \pm 0.9$ & $8.0 \pm 0.6^{*}$ \\
\hline
\end{tabular}

$m g / g d w t$, Milligrams per gram dry weight tissue.

$* p<0.05$ versus control muscle.

$\dagger p<0.05$ versus proximal region.

unstimulated control and chronically stimulated LDMs is presented in Table I. At $0.2 \mathrm{~Hz}$, peak tension development was significantly lower in the chronically stimulated muscle fibers. However, there was no difference in this parameter between control and chronically stimulated muscle fibers at higher stimulation frequencies. In the chronically stimulated muscle, the velocity of tension relaxation was lower at all stimulation frequencies compared with that in control muscle fibers. At increased stimulation frequencies, the time to peak tension was significantly increased in the chronically stimulated muscle fibers compared with that in control muscles. The change in the percent decline in the velocity of tension development from $0.2 \mathrm{~Hz}$ stimulation frequency was similar for both chronically stimulated and control skeletal muscle fibers (Fig. 2). In contrast, the degradation in peak tension development was significantly greater in the control LDM as opposed to that in the chronically stimulated muscle (Fig. 3). To address the possibility that regional differences in skeletal muscle fiber contractile function may exist, we undertook a subproject in which muscle fibers were harvested from both the middle region and distal region of the contralateral control and chronically stimulated LDMs of two pigs (Table II). Contractile performance of control muscle fibers was identical in both the middle and distal regions. In contrast, a 50\% reduction in the normalized peak tension development and velocity shortening was observed in muscle fibers taken 


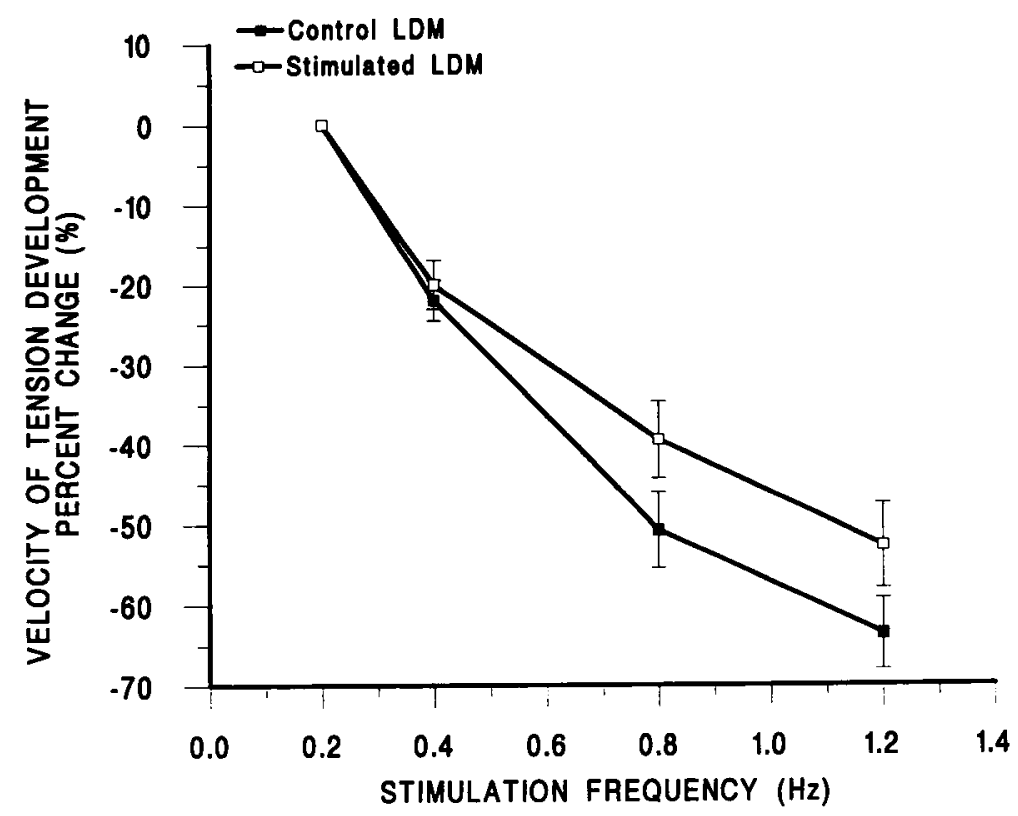

Fig. 2. Change in velocity of tension development as function of stimulation frequency for chronically stimulated LDM (Stimulated LDM) and contralateral control muscle (Control LDM). Similar decline in velocity of tension development was observed for both chronically stimulated and control skeletal muscle fibers.

from the distal region of the chronically stimulated LDM.

Skeletal muscle structure. Skeletal muscle fiber crosssectional area was determined from the proximal, middle, and distal regions of the contralateral control and chronically stimulated LDMs. A summary of these results is shown in Table III. The average cross-sectional area of muscle fibers taken at progressive distances from the LDM pedicle remained unchanged in control muscle. However, cross-sectional area of muscle fibers taken from the middle region of the chronically stimulated skeletal muscle significantly decreased from proximal region values. A further reduction in muscle fiber cross-sectional area was observed in sections taken from the distal portion of the chronically stimulated LDM. Representative electron micrographs taken from control and chronically stimulated LDMs are shown in Fig. 4. In control muscle, myofibril units were loosely organized within the muscle cell, and large areas of cytoplasmic space were easily visible (Fig. $4, A$ ). In the proximal and middle regions of chronically stimulated muscle, a dense distribution of myofibrils and mitochondria were clearly visible (Fig. 4, $B$ and $C$ ). The myofibril content appeared significantly reduced in sections examined from the distal region of chronically stimulated LDM (Fig. $4, D$ ). In these distal regions, the myofibril units appeared in disarray and were separated by wide cytoplasmic spaces. In addition, mitochondria exhibited high-amplitude swelling and vacuolization. A total of 970 electron micrographs taken from control muscles and 984 micrographs taken from chronically stimulated LDMs were subjected to quantitative morphologic study and the results are summarized in Table III. In the control muscle, there were no regional variations in myofibril content or mitochondrial density. In the chronically stimulated LDM, regional differences in both myofibril volumes and mitochondrial density were observed. In the proximal and middle regions of the chronically stimulated muscle, both myofibril and mitochondrial percent volume increased from control values. In the distal region of the chronically stimulated muscle, myofibril volume decreased from proximal region and middle region values and from contralateral control values. In contrast, mitochondrial volume percent was increased in the distal region of the chronically stimulated muscle, consistent with mitochondrial swelling and vacuolization.

Extracellular structure and composition. Circumferentially oriented, silver-stained sections of the LDM were examined at specific intervals from the pedicle to the distal section of the LDM. Representative photomicrographs of sections taken at progressive distances from the muscle pedicle are shown in Fig. 5. Examination of sections taken proximal $(0$ to $4 \mathrm{~cm})$ to the pedicle revealed a homogenous distribution of muscle cells surrounded by a fine band of collagenous matrix tissue (Fig. $5, C$ ). These bands of extracellular material divided the muscle cells into well-defined fascicles (Fig. 5,D). Sections taken from the middle region of the LDM ( 4 to $7 \mathrm{~cm}$ ) revealed focal 


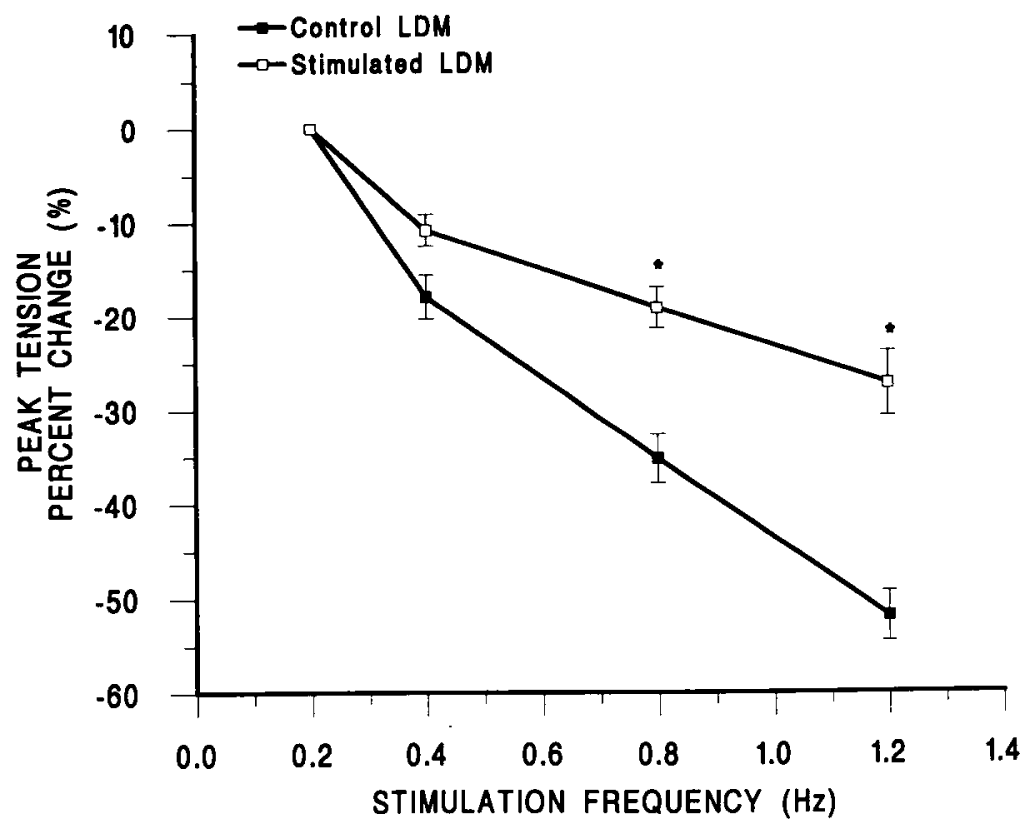

Fig. 3. Change in peak tension development as function of stimulation frequency. In contrast to velocity of tension development (Fig. 2), degradation in peak tension development was significantly higher in control LDM (Control $L D M$ ) as opposed to that in chronically stimulated muscle (Stimulated $L D M$ ). Thus chronic electrical stimulation of LDM resulted in fatigue resistance and improved muscle fiber contractile function at increased stimulation frequencies. Asterisk indicates $p<0.05$ versus control LDM.

areas of muscle cell loss with a thickening of the extracellular weave surrounding muscle fascicles (Fig. 5, $E$ and $F$ ). These zones of fibrosis became more frequent in sections taken from the distal region (more than $7 \mathrm{~cm}$ from the skeletal muscle pedicle). In these regions, widespread fibrosis with atrophy and dissolution of muscle cells was observed (Fig. 5, $G$ and $H$ ). Quantitative morphometry of the silver-stained sections was done and the results are summarized in Table III. A progressive increase in extracellular staining was observed in sections taken from the middle and distal regions of the chronically stimulated LDM. Biochemical measurements of hydroxyproline are summarized in Table III. Hydroxyproline content did not significantly change in any region of the control LDM (Table III). In the chronically stimulated muscle, hydroxyproline content was significantly increased from control values. Although hydroxyproline concentrations were higher in the middle and distal regions of the chronically stimulated muscle compared with those of the proximal region, these values did not reach statistical significance $(p=0.35)$.

\section{Discussion}

Changes in the composition of the muscle cell or the extracellular matrix may significantly influence LDM performance with dynamic cardiomyoplasty. Accordingly, the present study examined skeletal muscle structure and function after chronic electrical stimulation with dynamic cardiomyoplasty. To our knowledge, this is the first study that has examined the regional changes in LDM composition and structure in any model of dynamic cardiomyoplasty. After 6 weeks of dynamic cardiomyoplasty in pigs, the contractile function of isolated LDM fibers revealed slow twitch characteristics and fatigue resistance. The structure of the chronically stimulated muscle fibers revealed reduced cross-sectional area and increased myofibril content. However, contractile function of muscle fibers taken from the distal region of the chronically stimulated LDM was reduced. In the distal region of the chronically stimulated LDM, myofibril volume was decreased and collagen content increased.

Salmons and Streter ${ }^{8}$ and Salmons and Vrbova ${ }^{9} \mathrm{dem}-$ onstrated that skeletal muscle fibers could be converted from fast-twitch fibers to fatigue-resistant slow-twitch fibers with an electrical stimulation. With an electrical stimulation training protocol, these studies along with others have demonstrated that skeletal muscle fibers undergo changes in myosin adenosinetriphosphatase isoforms and force-development characteristics. $8,9,11,20$ In the present study, chronic electrical stimulation of the LDM in pigs resulted in skeletal muscle contractile function characteristics consistent with fatigue resistance. Furthermore, the proximal and middle regions of the chronically stimulated skeletal muscle showed structural 

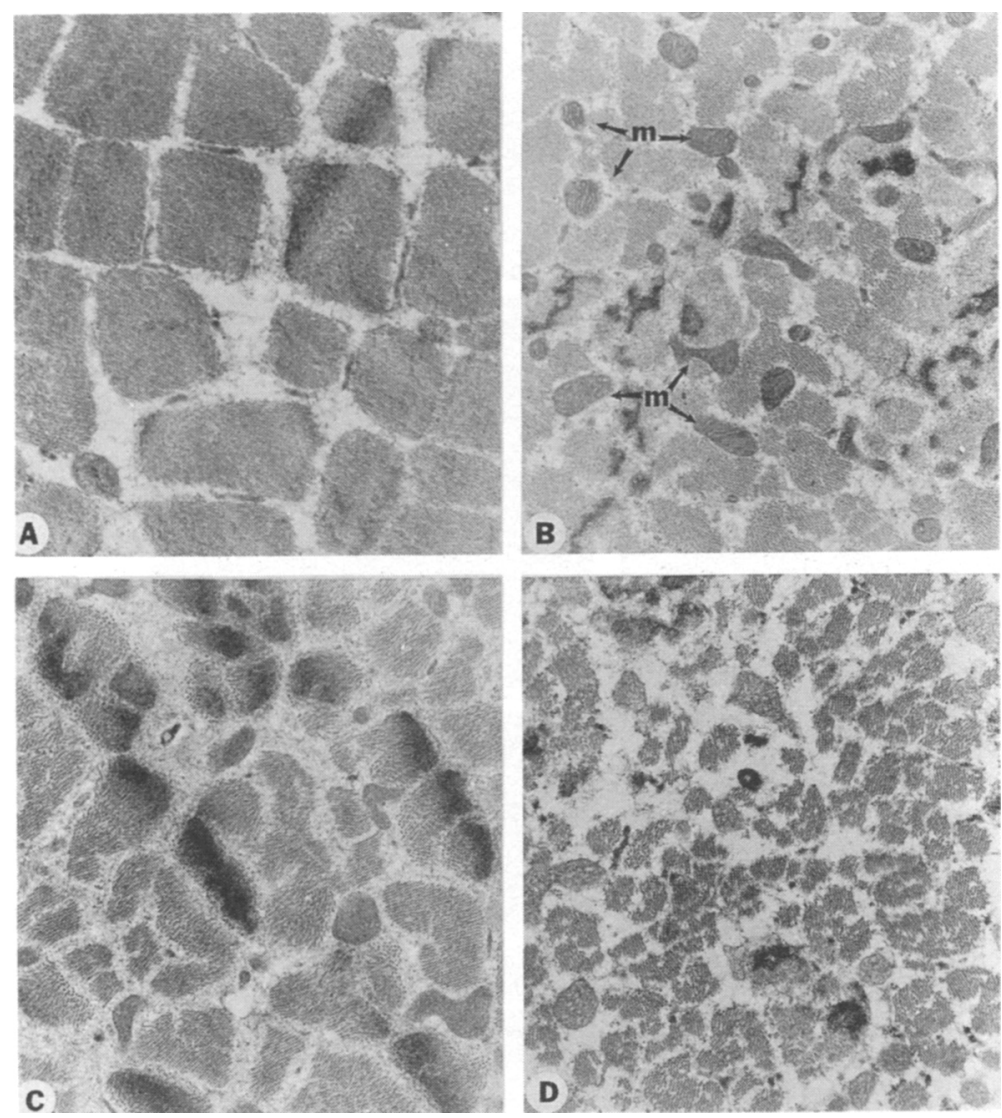

Fig. 4. Representative electron micrographs taken in cross section from unstimulated contralateral control LDM. A, In this control unstimulated muscle, loose organization of myofibrils can be observed with large amount of cytoplasmic space separating contractile units. B, In cross sections taken from proximal region of chronically stimulated muscle, densely packed myofibrils were observed with significantly increased number of mitochondria ( $m$, arrows). C, In middle region of chronically stimulated LDM, cytoplasmic areas between myofibrils were observed. Mitochondria were readily visible within cytoplasm surrounding these myofibrils. $\mathbf{D}$, In distal region of stimulated muscle, degeneration and myofibrillysis were readily observed within skeletal muscle cells. These ultrastructural changes are consistent with degenerative changes. Quantitative morphometry was done on electron micrographs obtained from each region of control and chronically stimulated skeletal muscle and results are summarized in Table III. (Original magnification $\times 10,000$.)

changes (increased myofibril and mitochondrial content) that are consistent with skeletal muscle transformation with electrical stimulation. ${ }^{8,9}$ However, in the present study, skeletal muscle structure and function in the distal region of the chronically stimulated muscle revealed dissimilar characteristics from those observed in the proximal and middle regions of the LDM. Whereas the reduction in muscle cell cross-sectional area may be an adaptive response in the proximal and middle regions of the chronically stimulated LDM, results from the present study suggest that the reduced muscle cell size in the distal segment of this skeletal muscle may actually be a degenerative change.

In a previous study, Mannion and associates ${ }^{11}$ exam- ined the induction of fatigue resistance in chronically stimulated LDM. In that report, isometric twitch recordings were obtained from chronically stimulated LDM and the contralateral control muscle. Fatigue resistance was indexed by measuring the decline in the peak developed tension as a function of time. These investigators found that the initial peak isometric tension developed and the fatigue rate of the trained muscles were significantly lower compared with those of the contralateral control muscle. In the present study, the isometric twitch properties of isolated muscle fibers taken from chronically stimulated and contralateral control LDMs were examined. Results from this present study are similar to those of the study of Mannion and associates ${ }^{11}$ in that the chronically 

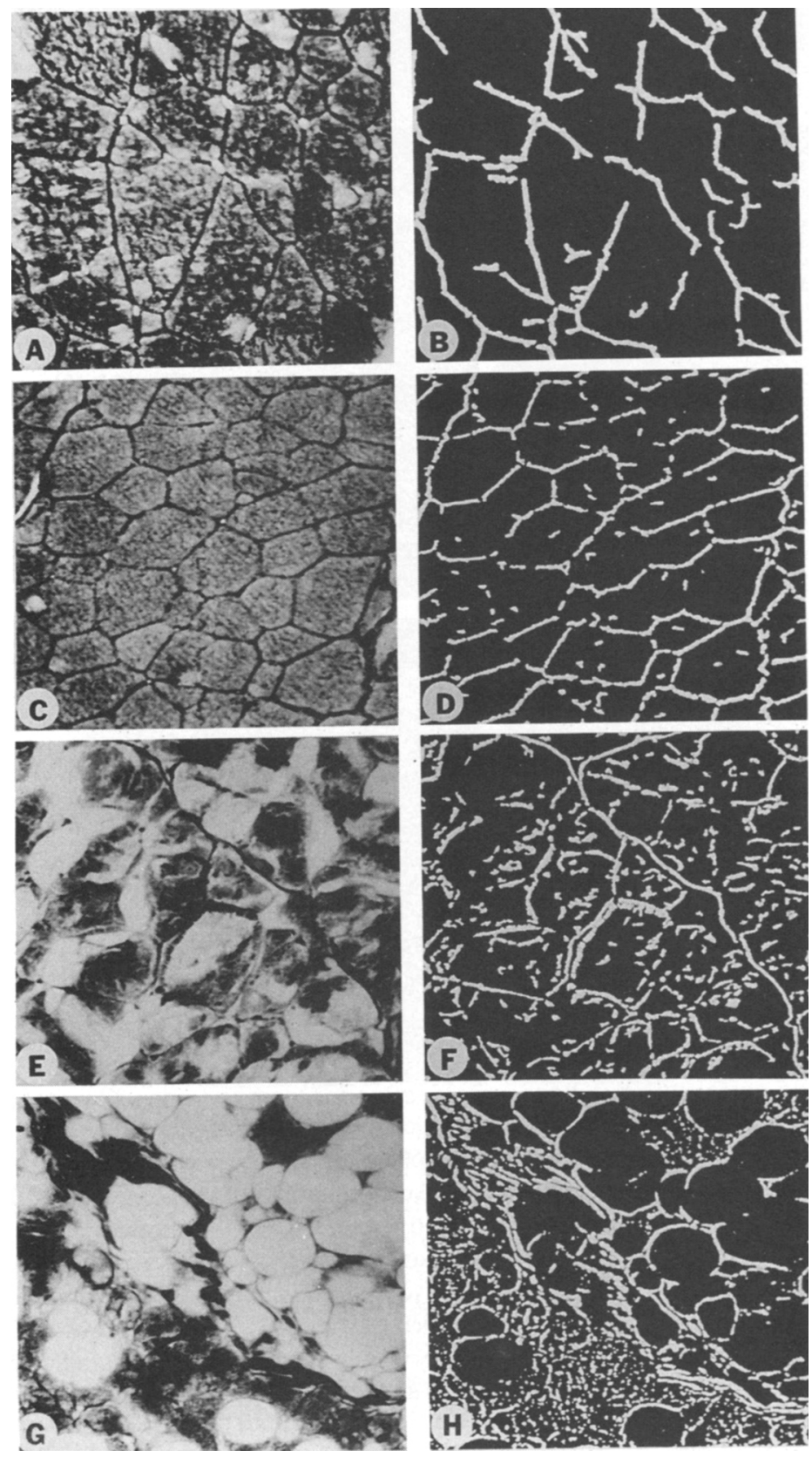

Fig. 5. Representative muscle sections taken from control and chronically stimulated LDMs after silver impregnation staining. Photomicrographs on left are phase-contrast photomicrographs. On right of each photomicrograph is displayed same image after input to image-analysis system and digital subtraction. By this method, percent area occupied by positive-staining extracellular material can be directly quantitated. A, Section taken from proximal region of contralateral control (unstimulated) LDM. Muscle fibers are in cross-sectional orientation and are separated by fine extracellular latticework. B, Digital image of photomicrograph shown in A revealed fine collagenous network surrounding individual muscle fibers. C, Section taken from proximal region of chronically stimulated LDM. Muscle fibers are in cross-sectional orientation and appear to have reduced cross-sectional diameter compared with unstimulated LDM. D, Digital image of this proximal section revealed increased abundance of extracellular material surrounding muscle cells. E, Representative photomicrograph of silver-stained section from middle region of chronically stimulated LDM. Increased vacuole formation was apparent within individual muscle cells. F, Digital subtraction of this image revealed increased extracellular material surrounding individual muscle cells. G, Representative photomicrograph of muscle section taken from distal region of chronically stimulated LDM. Large areas of muscle tissue appear to be replaced by fatty degeneration. Vacuole formation within existing muscle cells was clearly visible. H, Digital image revealed large chords of extracellular staining consistent with collagen fiber formation. (Original magnification of all photomicrographs $\times 250$.) 
stimulated muscle fibers exhibited fatigue resistance and prolonged contraction times compared with those of the contralateral control muscle. However, the present study builds on this past study by examining the relationship between changes in skeletal muscle function to changes in structure after chronic electrical stimulation and dynamic cardiomyoplasty. In the present study, chronic electrical stimulation of the LDM with dynamic cardiomyoplasty caused a reduction in skeletal muscle cell crosssectional area. In the report by Mannion and colleagues ${ }^{11}$ a similar directional reduction in muscle cell size was observed after 6 weeks of chronic LDM stimulation in dogs. In that report, muscle cell cross-sectional area decreased by approximately $30 \%$ compared with unstimulated control fibers. In the present study, cross-sectional muscle cell area not only declined after chronic electrical stimulation, but was also reduced in a region-specific manner. In the distal region of the chronically stimulated LDM, muscle cell cross-sectional area was reduced by more than $25 \%$ compared with the proximal and middle regions. The reduction in peak tension development in the chronically stimulated LDM may be caused in part by this reduction in muscle cell cross-sectional area.

In the present study, myofibril and mitochondrial percent volume within skeletal muscle cells increased in the proximal and middle regions of the chronically stimulated LDM and was accompanied by a reduction in myocyte cross-sectional area. Thus, on a per unit volume basis, chronic electrical stimulation of the LDM resulted in significantly increased contractile protein content and mitochondrial density. Past reports have suggested that the reduction in muscle cell diameter may be an adaptive response to continuous use. ${ }^{11,20}$ For example, a smaller fiber diameter will reduce the oxygen diffusion distance across the muscle cell. This may be of particular importance in the chronically stimulated muscle cell, which is more dependent on aerobic metabolism than it is under normal conditions. ${ }^{8,9,11,21}$ Past reports have shown that chronic electrical stimulation of the LDM causes a predominance of slow myosin isoenzymes, changes in myofibrillar adenosinetriphosphatase activity, and increased oxidative phosphorylation. ${ }^{21}$ The increased myofibril content observed in the present study was accompanied by a significant increase in mitochondrial volume. These results suggest that the structural basis for the increased oxidative phosphorylation capacity observed in past reports $^{21}$ is a result of increased mitochondrial volume within the chronically stimulated muscle cells.

The extracellular matrix, a three-dimensional collagen latticework, is essential for coordinated force transmission and maintenance of skeletal muscle geometry and alignment. With dynamic cardiomyoplasty, the LDM must maintain its conformation around the ventricular chambers in order for appropriate force transmission to occur. Alterations in the extracellular matrix may result in changes in the skeletal muscle geometry that will significantly affect the degree of force delivery to the ventricular chamber. In the present study, significant changes within the extracellular matrix occurred within the LDM after 6 weeks of chronic electrical stimulation. Furthermore, these changes within the extracellular space occurred in a progressive fashion, from the proximal to the distal region of the skeletal muscle. With mobilization of the LDM and chronic electrical stimulation, a significant increase in collagen content was observed. In the proximal regions, an increased collagenous weave was observed to surround individual muscle cells. This collagenous weave increased in the muscle sections taken from the middle region of the chronically stimulated LDM. In a past report in which a similar stimulation protocol was used, Mannion and colleagues ${ }^{11}$ reported a similar qualitative increase in connective tissue. In the present study, the changes within the extracellular space in the distal region of the chronically stimulated LDM were in marked contrast to findings in the proximal and middle regions. The geometric distribution and structure of the extracellular matrix were significantly different in the distal region compared with the more proximal regions of the chronically stimulated LDM.

The mechanisms responsible for the region-specific changes in skeletal muscle structure with chronic electrical stimulation of the LDM are probably a result of alterations in blood flow, abnormalities in metabolism, or both. Mobilization of the LDM for creation of the cardiomyoplasty results in disruption of blood flow to the regions most distal to the thoracodorsal neurovascular pedicle. ${ }^{1-10}$ In a report by Mannion and colleagues, ${ }^{22}$ disruption of the collateral blood supply of the LDM in dogs resulted in significantly reduced blood flow to the distal segment of the muscle. Thus a probable contributory mechanism for the reduced myofibril content and muscle cell size and increased fibrous weave observed in the distal regions of the chronically stimulated LDM is reduced blood flow. In these distal regions, increased vacuole formation was observed within the cell and within the mitochondria. It is well-established that chronically stimulated skeletal muscle has increased oxidative phosphorylation capacity and is dependent on aerobic metabolism. 8, 9, 20,21 Thus the appearance of these vacuoles, particularly associated with mitochondria, may be a result of a disruption in aerobic metabolism. On the basis of the findings from the present study, future studies aimed at more careful examination of regional changes in blood flow and metabolism would seem appropriate. 


\section{Summary}

Dynamic cardiomyoplasty has been demonstrated to provide symptomatic relief in patients with severe congestive heart failure. ${ }^{1-7}$ In clinical studies performed worldwide, chronic stimulation of the LDM in synchrony with the intrinsic ventricular contraction has been shown to improve left ventricular pump function. ${ }^{1-7}$ However, for this surgical treatment of congestive heart failure to gain wider application and acceptance, the structural and functional events that occur within the chronically stimulated skeletal muscle must be understood. In the present study, regional changes in cellular and extracellular structure were observed after chronic electrical stimulation of the LDM with dynamic cardiomyoplasty. These regional changes in skeletal muscle structure were associated with regional changes in skeletal muscle contractile function. These region-specific changes in LDM structure and function may significantly influence the long-term performance of this skeletal muscle with dynamic cardiomyoplasty. Future studies directed at improving the regional alterations in muscle structure with chronic electrical stimulation and dynamic cardiomyoplasty may be appropriate.

\section{REFERENCES}

1. Magovern GJ, Christlieb IY, Kao RL. The Allegheny Hospital experience. In: Carpentier A, Chachques JC, Grandjean P, eds. Cardiomyoplasty. New York: Futura, 1991:159-70.

2. Magovern JA, Furnary AP, Christlieb IY, Kao RL, Magovern GJ. Right latissimus dorsi cardiomyoplasty for left ventricular failure. Ann Thorac Surg 1992;53:1120-2.

3. Moreira LFP, Seferian P Jr, Bocchi EA, et al. Survival improvement with dynamic cardiomyoplasty in patients with dilated cardiomyopathy. Circulation 1991;84(Suppl):III296-302.

4. Carpentier A, Abry B, Mihaileanu S, Chachques JC. Hopital Broussais Clinical experience. In: Carpentier A, Chachques JC, Grandjean P, eds. Cardiomyoplasty. New York: Futura, 1991:147-57.

5. Chachques JC, Grandjean P, Schwartz K, et al. Effect of latissimus dorsi dynamic cardiomyoplasty on ventricular function. Circulation 1988;78(Suppl):III203-16.

6. Magovern GJ, Heckler FR, Park SB, et al. Paced skeletal muscle for dynamic cardiomyoplasty. Ann Thorac Surg 1988;45:614-9.

7. Salmons S, Jarvis JC. Skeletal muscle as an adaptive contractile biomaterial for cardiac assistance: fundamental considerations. In: Morucci JP, Plonsey R, Coatrieux JL, Laxminarayan S, eds. Proceedings of the 14th annual conference of the IEEE Engineering in Medicine and Biology
Society, Paris, France, October 29-November 1, 1992. Piscataway, NJ: IEEE, 1992:2798-9.

8. Salmons S, Streter FA. Significance of impulse activity in the transformation of skeletal muscle type. Nature 1976; 263:30-4.

9. Salmons S, Vrbova G. The influence of activity on some contractile characteristics of mammalian fast and slow muscle. J Physiol 1969;201:535-49.

10. Carpentier A, Chachques JC. Cardiomyoplasty: surgical technique. In: Carpentier A, Chachques JC, Grandjean P, eds. Cardiomyoplasty. New York: Futura, 1991:105-122.

11. Mannion JD, Bitto T, Hammond RL, Rubinstein NA, Stephenson LW. Histochemical and fatigue characteristics of conditioned canine latissimus dorsi muscle. Circ Res 1986;58:298-304.

12. Institute of Laboratory Animal Resources, Committee on Care and Use of Laboratory Animals. Guide for the care and use of laboratory animals. National Research Council. National Institutes of Health. NIH publication 86-23, revised 1985 .

13. Spinale FG, Zellner JL, Tomita M, Crawford FA, Zile MR. Relationship between ventricular and myocyte remodeling with the development and regression of supraventricular tachycardia-induced cardiomyopathy. Circ Res 1991;69:1058-67.

14. Spinale FG, Tomita M, Zellner JL, Cook JC, Crawford FA, Zile MR. Collagen remodeling and changes in LV function during development and recovery from supraventricular tachycardia. Am J Physiol 1991;261:H308-18.

15. Gordon AM, Huxley F, Julian FJ. Tension development in highly stretched vertebrate muscle fibres. J Physiol 1966; 184:143-69.

16. Stegemann H, Stalder K. Determination of hydroxyproline. Clin Chim Acta 1967;18:267-73.

17. Steel RGD, Torrie JH, eds. Principles and procedures of statistics: a biometrical approach. New York: McGrawHill, 1980:533-53.

18. Steel RGD, Torrie JH, eds. Principles and procedures of statistics: a biometrical approach. New York: McGrawHill, 1980:469-76.

19. Steel RGD, Torrie JH, eds. Principles and procedures of statistics: a biometrical approach. New York: McGrawHill, 1980:172-94.

20. Ciske PE, Faulkner JA. Chronic electrical stimulation of nongrafted and grafted skeletal muscles in rats. J Appl Physiol 1985;59:1434-9.

21. Clark BJ III, Acker MA, McCully $\mathrm{K}$, et al. In vivo ${ }^{31} \mathrm{P}-\mathrm{NMR}$ spectroscopy of chronically stimulated canine skeletal muscle. Am J Physiol 1988;254:C258-66.

22. Mannion JD, Velchick M, Hammond R, et al. Effects of collateral blood flow vessel ligation and electrical conditioning on blood flow in dog latissimus dorsi muscle. J Surg Res 1989;47:332-40. 